\title{
Discrete-Time Filter Synthesis Using Product of Gegenbauer Polynomials
}

\author{
Nikola STOJANOVIC ${ }^{1}$, Negovan STAMENKOVIC ${ }^{2}$ Ivan KRSTIC $^{3}$ \\ ${ }^{1}$ University of Niš, Faculty of Electronics, A. Medvedeva 14, 18000 Niš, Serbia \\ ${ }^{2}$ University of Priština, Faculty of Natural Science and Mathematics, 38220 K. Mitrovica, Serbia \\ ${ }^{3}$ University of Priština, Faculty of Technical Sciences, 38220 K. Mitrovica, Serbia \\ nikola.stojanovic@elfak.ni.ac.rs,negovan.stamenkovic@pr.ac.rs, ivan.krstic@pr.ac.rs
}

Manuscript received May 23, 2016

\begin{abstract}
A new approximation for designing continuoustime and discrete-time low-pass filters, presented in this paper, based on the product of Gegenbauer polynomials, provides the ability of more flexible adjustment of passband and stopband responses. The design is achieved taking into account a prescribed specification, leading to a better trade-off among the magnitude and group delay responses. Many wellknown continuous-time and discrete-time transitional filter based on the classical polynomial approximations (Chebyshev, Legendre, Butterworth) are shown to be special cases of proposed approximation method.
\end{abstract}

\section{Keywords}

Gegenbauer polynomials, lowpass filters, continuoustime filters, discrete-time filters

\section{Introduction}

The high-performance lowpass filter should exhibit high selectivity, low passband insertion loss, and small group delay variations in the passband. As these requirements are contradictory, a lowpass filter is often optimized for one of these parameters by using some of the well-known approximation methods: Chebyshev, Butterworth or Bessel among others. Chebyshev filter [1] exhibits higher selectivity than Butterworth filter but also has ripples and significant variations of group delay in the passband. On the contrary, Bessel filter exhibits maximally flat group delay but very low selectivity

A particular class of the all-pole filters includes filters derived from the product of two orthogonal polynomial components such as products of Legendre polynomials [2], product of Chebyshev polynomials [3], transitional ButterworthChebyshev [4], [5], product of Chebyshev of the first kind and Chebyshev of the second kind [6], Butterworth-Legendre [7], etc. Filter synthesis using product of orthogonal Gegenbauer (ultraspherical) [8] polynomials is proposed in this paper.
The idea to use prototypes derived in this way comes from the consideration that they exhibit continuous behavior between Chebyshev and Butterworth prototypes, via the abovementioned prototypes derived from products of orthogonal polynomials.

The discrete-time filter design is based on expressing the squared magnitude function $\left|H\left(e^{-j \Omega}\right)\right|^{2}$ as a rational function of $\tan (\Omega / 2)$ or $\sin (\Omega / 2)$, where $\Omega$ is digital angular frequency in radians-per-sample ${ }^{1}$. Filters designed using this approach are popularly known as the tangent type and the sine type of filters or simpler tan and sine filters [9]. A new family of continuous-time and discrete-time filters, termed as product of Gegenbauer polynomials, has been proposed, and their performances analyzed.

\section{Approximation}

In general, the squared magnitude response of the lowpass prototype filter transfer function is represented as:

$$
H_{n}^{2}(x)=\frac{1}{1+\varepsilon^{2} \phi_{n}^{2}(x)}
$$

where $x$ is a real frequency variable, $\phi_{n}(x)$ is the approximating or characteristic function of the lowpass filter which at the passband edge holds $\phi_{n}(1)=1$ and $\varepsilon$ is a design parameter related to the maximal passband attenuation $a_{\max }$ (in $\mathrm{dB}$ ) as $\varepsilon=\sqrt{10^{0.1 a_{\max }}-1}$. Using (1), we introduce a new filters approximation with the characteristic function composed as the product of two Gegenbauer polynomials:

$$
\phi_{n}(x)=\frac{C_{k}^{\nu_{1}}(x)}{C_{k}^{\nu_{1}}(1)} \frac{C_{n-k}^{\nu_{2}}(x)}{C_{n-k}^{v_{2}}(1)}
$$

where $C_{k}^{v}(x)$ is a Gegenbauer polynomial [10] of order $v>-0.5$ and degree $k, n$ is the filter's degree, $k$ is the interpolator factor, and:

$$
C_{k}^{v}(1)=\left(\begin{array}{c}
k+2 v-1 \\
k
\end{array}\right)=\frac{\Gamma(k+2 v)}{\Gamma(k+1) \Gamma(2 v)}
$$

\footnotetext{
$\left.{ }^{1} \Omega\right|_{\mathrm{rad} / \text { sample }}=\left.f\right|_{\mathrm{Hz}} \frac{2 \pi}{f_{s}}$, where $f_{S}$ is sampling frequency in $\mathrm{Hz}$.
} 
where $\Gamma(x)$ is well known gamma function. Note that for $k=0$ filter presents $C_{n}^{\nu_{2}}(x)$, for $k=n$ filter presents $C_{n}^{\nu_{1}}(x)$, and for $0 \leq k \leq n$ the filter presents mixture of $C_{k}^{\nu_{1}}(x)$ and $C_{n-k}^{\nu_{2}}(x)$ components.

The desired transfer function can be generated from (1) by analytic continuation to the complex domain and selecting poles so that transfer function is stable.

Five degrees of freedom are available for the filter design by using the product of Gegenbauer polynomials: the filter degree $n$, the interpolator factor $k$, design parameter $\varepsilon$, and orders of Gegenbauer polynomials, $v_{1}$ and $v_{2}$.

\section{Continuous-Time Filters}

The continuous-time transfer functions $H_{n}(s)$ are determined from $H_{n}^{2}(x)$ by replacing frequency variable $x$ with $x=-\mathrm{j} s$. At real frequencies is $s=\mathrm{j} \omega$. The squared magnitude function (1) is transformed into:

$$
\left.H_{n}^{2}(x)\right|_{x=-\mathrm{j} s}=H_{n}(s) H_{n}(-s) .
$$

The poles of (4) are zeros of the following equation:

$$
1+\left.\varepsilon^{2}\left[\frac{C_{k}^{\nu_{1}}(x)}{C_{k}^{\nu_{1}}(1)} \frac{C_{n-k}^{\nu_{2}}(x)}{C_{n-k}^{\nu_{2}}(1)}\right]^{2}\right|_{x=-\mathrm{j} s}=0 .
$$

The continuous-time transfer functions $H_{n}(s)$ have to be stable with all their poles lying in the left half of the $s$-plane. Symbolic toolbox package from Matlab can be used to find all zeros of the polynomial (5).

The transfer function of the proposed filter has one of the following forms:

$$
H_{n}(s)=\frac{h_{0}}{\prod_{i=0}^{n-1}\left(s-s_{i}\right)}=\frac{h_{0}}{\sum_{i=0}^{n} a_{i+1} s^{n-i}}=\frac{1}{\sum_{i=0}^{n} d_{i+1} s^{n-i}}
$$

where $s_{i}$ are poles of the transfer function $H_{n}(s)$ and $h_{0}=a_{n+1} \sqrt{H_{n}^{2}(0)}$ is constant which ensures that magnitude response $H_{n}(\omega)$ is bounded above by unity. If $n$ is odd at the zero-frequency the characteristic function is also equal to zero, that is $H_{n}(0)=1$ which gives $h_{0}=a_{n+1}$.

For filters considered here, the comparison of slope steepness at the cutoff frequency (cutoff slope) can be determined by calculating slope of the magnitude function taken with negative sign at the passband edge, defined by $S_{\mathrm{ct}}=-|\mathrm{d}| H_{n}(\mathrm{j} \omega) /\left.\mathrm{d} \omega\right|_{\omega=1}$. Denoting the cutoff slope of the magnitude frequency response (1) at $\omega=1$ by $S_{\mathrm{ct}}$, we have:

$$
S_{\mathrm{ct}}=2^{-\frac{3}{2}}\left|\frac{1}{C_{k}^{\nu_{1}}(1)} \frac{\mathrm{d} C_{k}^{\nu_{1}}(\omega)}{\mathrm{d} \omega}+\frac{1}{C_{n-k}^{\nu_{2}}(1)} \frac{\mathrm{d} C_{n-k}^{\nu_{1}}(\omega)}{\mathrm{d} \omega}\right|_{\omega=1}
$$

where $\varepsilon=1$ and

$$
\left.\frac{\mathrm{d} C_{j}^{\nu}(\omega)}{\mathrm{d} \omega}\right|_{\omega=1}=2 v C_{j-1}^{\nu+1}(1), \quad j=k \quad \text { or } n-k .
$$

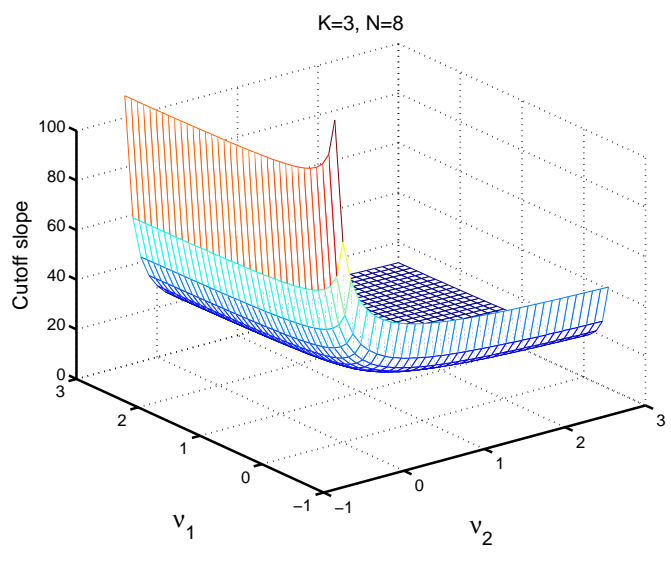

Fig. 1. Cutoff slope contour plot of the eight-degree filter derived from product of Gegenbauer polynomials with $k=3$.

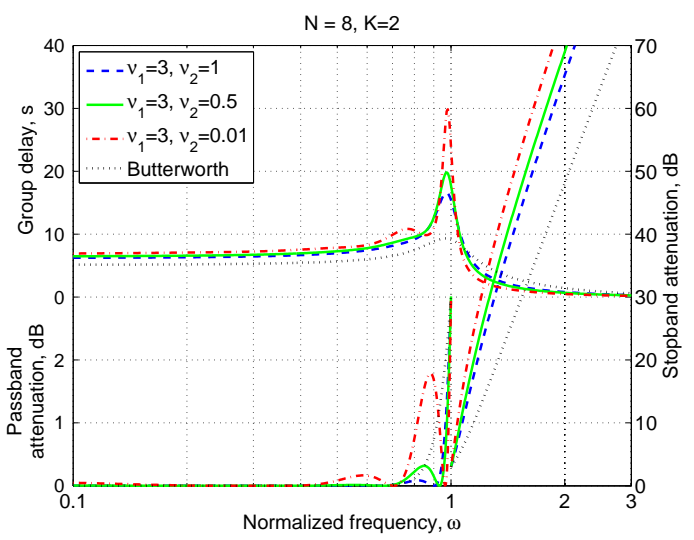

Fig. 2. Frequency responses of the proposed filters. by:

The cutoff slope obtained by putting (8) into (7) is given

$$
S_{\mathrm{ct}}=2^{-\frac{3}{2}}\left[\frac{k\left(k+2 v_{1}\right)}{2 v_{1}+1}+\frac{(n-k)\left(n-k+2 v_{2}\right)}{2 v_{2}+1}\right] \text {. }
$$

In Fig. 1, the three-dimensional plot of the cutoff slope of the eight-degree filter obtained using the product of two Gegenbauer polynomials with $k=3$ is given.

As an illustration, the frequency responses of continuous-time lowpass filters designed by proposed method are plotted in Fig. 2 for $n=8, k=2, v_{1}=3$ and various values of $v_{2}$. In all cases the $3 \mathrm{~dB}(\varepsilon=1)$ cutoff frequency is $\Omega_{\mathrm{c}}=1$.

Finally, in order to facilitate the practical application of these filter functions, the denominator coefficients of the continuous-time transfer functions $\left(\varepsilon=1, k=2, v_{1}=3\right.$ and $v_{2}=0.5$ ) derived from (5) and (6) for $n=4$ to 10 are presented in Tab. 1. In all cases the frequency is normalized so that $\omega_{3 \mathrm{~dB}}=1$. Pascal matrix is a numerical algorithm by means of which it is possible to transform the coefficients of continuous-time filters (given in Tab. 1) to the coefficients of discrete-time filters [11]. Synthesis of the discrete-time filters directly in the $z$-domain is given in the following section. 


\begin{tabular}{|c|c|c|c|c|c|c|c|c|c|c|c|}
\hline \multirow[b]{2}{*}{$n$} & \multicolumn{11}{|c|}{$D(s)=d_{1} s^{n}+d_{2} s^{n-1}+\cdots+d_{n} s+d_{n+1}$} \\
\hline & $d_{1}$ & $d_{2}$ & $d_{3}$ & $d_{4}$ & $d_{5}$ & $d_{6}$ & $d_{7}$ & $d_{8}$ & $d_{9}$ & $d_{10}$ & $d_{11}$ \\
\hline 4 & 1.7143 & 3.3376 & 4.0347 & 2.8245 & 1.0025 & & & & & & \\
\hline 5 & 2.8571 & 5.8054 & 7.9694 & 8151 & 3.6981 & 1.0000 & & & & & \\
\hline 6 & 5.0000 & 10.5041 & 15.9443 & 15.5406 & 10.6017 & 4.5968 & 1.0014 & & & & \\
\hline 7 & 9.0000 & 19.4225 & 32.0824 & 34.4486 & 27.4285 & 15.2518 & 5.5295 & 1.0000 & & & \\
\hline 8 & 16.5000 & 36.4136 & 64.7428 & 75.0433 & 67.1877 & 44.0691 & 20.9217 & 6.4629 & 1.0010 & & \\
\hline 9 & 30.6429 & 68.9289 & 130.8556 & 161.5178 & 159.0185 & 117.8603 & 66.9725 & 27.4717 & 7.4190 & 1.0000 & \\
\hline 10 & 57.4554 & 131.4054 & 264.6998 & 344.5520 & 367.5141 & 299.9290 & 194.8424 & 96.4683 & 35.0584 & 8.3688 & 1.0008 \\
\hline
\end{tabular}

Tab. 1. Coefficients of filters derived from the product of Gegenbauer polynomials $C_{2}^{3}(\omega) C_{n-2}^{0.5}(\omega)$.

\section{Discrete-Time Filters}

Discrete-time filters are specified by its squared magnitude function using procedure akin to continuous-time one. Accordingly, the corresponding discrete-time filter's magnitude function can be obtained from the magnitude response (1) by replacing $x$ with $\sin (\Omega / 2) / \sin \left(\Omega_{c} / 2\right)$ for the sine filter and with $\tan (\Omega / 2) / \tan \left(\Omega_{\mathrm{c}} / 2\right)$ for the $\tan$ filter [7], [12], where $\Omega$ is discrete-time frequency, and $\Omega_{\mathrm{c}}$ discrete-time cutoff frequency. In the first case the so-called discrete-time sine filter or all-pole filter is obtained, whereas the second case gives the discrete-time tan filter or pole-zero filter [7], [12].

\subsection{Sine Filters}

The discrete-time sine transfer functions $H_{n}(z)$ are obtained from squared magnitude function (1) by replacing frequency variable $x$ with $x=-\mathrm{j}(z-1) /(2 \beta \sqrt{z})$, where $z=e^{\mathrm{j} \Omega}$ at the real frequencies. Thus, for the sine filters we have:

$$
\left.H_{n}^{2}(x)\right|_{x=-\mathrm{j} \frac{1}{\beta} \frac{z-1}{2 \sqrt{z}}}=H_{n}(z) H_{n}(1 / z)
$$

The poles of (10) are zeros of the following equation:

$$
1+\left.\varepsilon^{2}\left[\frac{C_{k}^{\nu_{1}}(x)}{C_{k}^{\nu_{1}}(1)} \frac{C_{n-k}^{\nu_{2}}(x)}{C_{n-k}^{\nu_{2}}(1)}\right]^{2}\right|_{x=-\mathrm{j} \frac{1}{\beta} \frac{z-1}{2 \sqrt{z}}}=0
$$

where $\beta=\sin \left(\Omega_{\mathrm{c}} / 2\right)$. Therefore, by finding all zeros of (11) and selecting poles inside the unit circle in the $z$-plane, $H(z)$ is obtained. Poles that lie outside the unit circle corresponds to $H(1 / z)$. This method leads to the design of all-pole (polynomial) discrete-time filter :

$$
H_{n}(z)=\frac{h}{1+a_{1} z^{-1}+\cdots+a_{n-1} z^{-n+1}+a_{n} z^{-n}}
$$

where $h=\sqrt{\left|H_{n}(x)\right|_{x=0}^{2}}\left(1+\sum_{i=1}^{n} a_{i}\right)$. This system has $n$-th order zero at $z=0$. Notice that any sine transfer function $H_{n}(z)$ of degree $n$ can be implemented with only $n+1$ multipliers and $n$ adders with two inputs and one output.

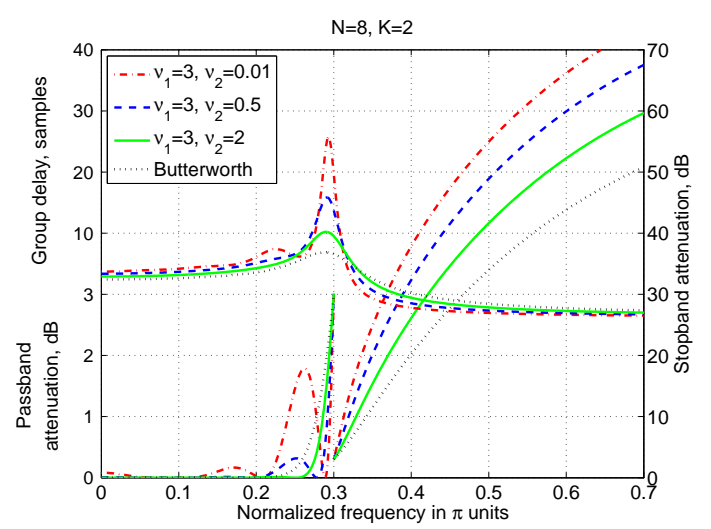

Fig. 3. Frequency responses of the proposed sine filters.

\begin{tabular}{crrr}
\hline Coeff. & \multicolumn{1}{c}{$v_{2}=0.01$} & \multicolumn{1}{c}{$v_{2}=0.5$} & \multicolumn{1}{c}{$v_{2}=2$} \\
\hline$a_{1}$ & -5.3207216 & -4.9242926 & -4.4063349 \\
$a_{2}$ & 13.4121170 & 11.5588036 & 9.3351183 \\
$a_{3}$ & -20.6660404 & -16.6244965 & -12.1505060 \\
$a_{4}$ & 21.1314583 & 15.8754463 & 10.5017481 \\
$a_{5}$ & -14.6079473 & -10.2432365 & -6.1246138 \\
$a_{6}$ & 6.6421704 & 4.3415184 & 2.3409913 \\
$a_{7}$ & -1.8110809 & -1.1014919 & -0.5340554 \\
$a_{8}$ & 0.2262488 & 0.1277661 & 0.0555098 \\
\hline$h$ & 0.0061459 & 0.0100078 & 0.0178570 \\
\hline
\end{tabular}

Tab. 2. Polynomial coefficients for the eight degree sine filters based on the product of Gegenbauer polynomials.

As an illustration, the frequency responses of the sine lowpass filters designed using this method are plotted in Fig. 3 for $n=8, k=2, v_{1}=3$ and various values of $v_{2}$. In all cases the $3 \mathrm{~dB}$ cutoff frequency is $\Omega_{\mathrm{c}}=0.3 \pi$. Corresponding coefficients of the eight degree transfer function, whose frequency responses are displayed in Fig. 3, are given in Tab. 2.

Cutoff slope of the proposed sine filters is given by:

$$
S_{\mathrm{d} t}^{\sin }=\left.S_{\mathrm{ct}} \frac{\mathrm{d}}{\mathrm{d} \Omega} \frac{\sin (\Omega / 2)}{\sin \left(\Omega_{\mathrm{c}} / 2\right)}\right|_{\Omega=\Omega_{\mathrm{c}}}=S_{\mathrm{ct}} \frac{1}{2 \tan \left(\Omega_{\mathrm{c}} / 2\right)}
$$

where $S_{\mathrm{ct}}$ is given by (9). The cutoff slope of the sine filter is steeper than that of the corresponding continuous-time filter, in the range $0<\Omega_{\mathrm{c}}<0.2952 \pi$, but less than in the range $0.2952 \pi<\Omega_{\mathrm{c}}<\pi$. 


\begin{tabular}{crrr}
\hline Coeff. & \multicolumn{1}{c}{$v_{2}=0.01$} & \multicolumn{1}{c}{$v_{2}=0.5$} & \multicolumn{1}{c}{$v_{2}=2$} \\
\hline$a_{1}$ & -5.1821237 & -4.7691240 & -4.2156720 \\
$a_{2}$ & 12.8581362 & 10.9932613 & 8.7220345 \\
$a_{3}$ & -19.6069736 & -15.6234627 & -11.1696520 \\
$a_{4}$ & 19.9167213 & 14.8075218 & 9.5507011 \\
$a_{5}$ & -13.7207289 & -9.5154219 & -5.5327225 \\
$a_{6}$ & 6.2357187 & 4.0292683 & 2.1079929 \\
$a_{7}$ & -1.7047225 & -1.0244730 & -0.4809017 \\
$a_{8}$ & 0.2142912 & 0.1194846 & 0.0501430 \\
\hline$h$ & 0.0000399 & 0.0000666 & 0.0001247 \\
\hline
\end{tabular}

Tab. 3. Polynomial coefficients for the 8th degree tan filters based on the product of Gegenbauer polynomials.

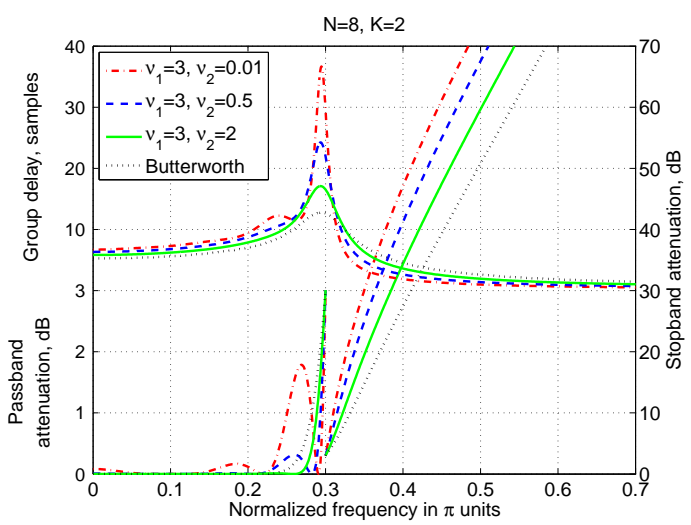

Fig. 4. Frequency responses of the proposed tan filters.

\subsection{Tan Filters}

The discrete-time tan transfer functions are obtained from the squared magnitude function (1) by replacing frequency variable $x$ with $x=-\mathrm{j}(z-1) /(\alpha(z+1))$, where $z=e^{\mathrm{j} \Omega}$ at the real frequencies. Thus, for the tan filters we use transformation:

$$
\left.H_{n}^{2}(x)\right|_{x=-\mathrm{j} \frac{1}{\alpha} \frac{z-1}{z+1}}=H_{n}(z) H_{n}(1 / z),
$$

known as the bilinear transformation. The poles of (14) are the zeros of the following equation:

$$
1+\left.\varepsilon^{2}\left[\frac{C_{k}^{\nu_{1}}(x)}{C_{k}^{\nu_{1}}(1)} \frac{C_{n-k}^{\nu_{2}}(x)}{C_{n-k}^{\nu_{2}}(1)}\right]^{2}\right|_{x=-\mathrm{j} \frac{1}{\alpha} \frac{z-1}{z+1}}=0
$$

where $\alpha=\tan \left(\Omega_{\mathrm{c}} / 2\right)$. Therefore, by finding zeros of (15) and by selecting the ones that lie inside the unit circle, $H(z)$ can be obtained. This method leads to the design of the pole-zero discrete-time filter which has a zero of order $n$ at $z=-1$ :

$$
H_{n}(z)=\frac{h\left(1+z^{-1}\right)^{n}}{1+a_{1} z^{-1}+\cdots+a_{n-1} z^{-n+1}+a_{n} z^{-n}} .
$$

where $h=\sqrt{\left|H_{n}(x)\right|_{x=0}^{2}}\left(1+\sum_{i=1}^{n} a_{i}\right) / 2^{n}$. This transfer function requires $2 n+1$ multipliers and $n$ three-input and one output adders (carry-save 3-to-2 adder followed by a standard full-adder) for implementation.

As an illustration, the frequency responses of the tan lowpass filters, designed using this method, are plotted in Fig. 4 for $n=8, k=2, v_{1}=3$ and various values of $v_{2}$. In all cases the $3 \mathrm{~dB}$ cutoff frequency is $\Omega_{\mathrm{c}}=0.3 \pi$.

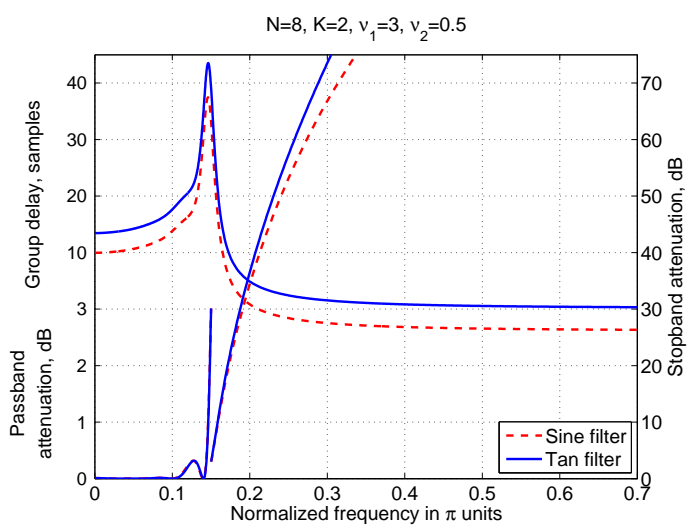

Fig. 5. Frequency responses comparison of tan and sine narrowband lowpass filters of degree 8 .

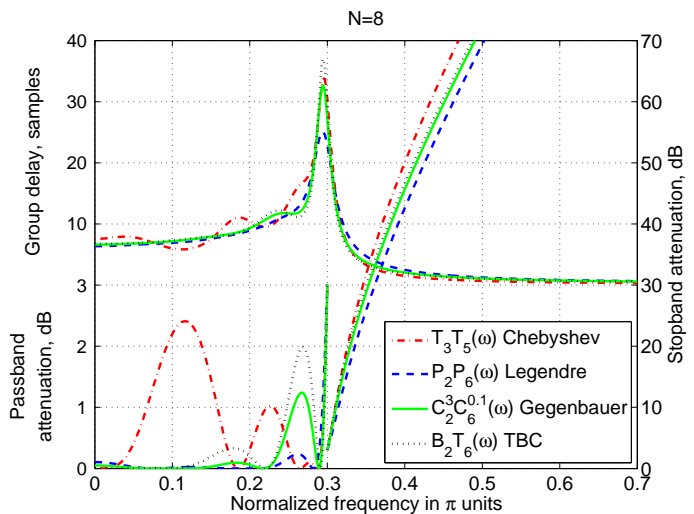

Fig. 6. Comparison of frequency responses of proposed filter and filters derived from products of the other orthogonal polynomials.

Corresponding coefficients of the eight-degree transfer function whose frequency responses are displayed in Fig. 4 are given in Tab. 3.

At the cutoff frequency $\Omega=\Omega_{\mathrm{c}}$, the discrete-time tan filters magnitude response slope can be calculated as:

$$
S_{\mathrm{d} t}^{\mathrm{tan}}=\left.S_{\mathrm{ct}} \frac{\mathrm{d}}{\mathrm{d} \Omega} \frac{\tan (\Omega / 2)}{\tan \left(\Omega_{\mathrm{c}} / 2\right)}\right|_{\Omega=\Omega_{\mathrm{c}}}=S_{\mathrm{ct}} \frac{1}{\sin \left(\Omega_{\mathrm{c}}\right)} .
$$

Since $0<\Omega_{\mathrm{c}}<\pi$, that is $\sin \left(\Omega_{\mathrm{c}}\right) \leq 1$ it can be concluded that the tan filter has steeper or equal cutoff slope than the corresponding continuous-time counterpart.

\section{Results of Approximation}

In applications where high ripple values in the passband are not tolerable, one could use filter derived from the product of Gegenbauer polynomials of degrees $k=2$ and $n-2$ with orders $v_{1}=0.5$ and $v_{2}=0.1$ respectively. If $n=7$ proposed filter has a small hump of about $0.0576 \mathrm{~dB}$ in the passband magnitude response, better group delay characteristic compared to Chebyshev filter and a higher cutoff slope than the Butterworth filter.

When $\Omega_{\mathrm{c}} \ll 1$, from (13) and (17), we have that $S_{\mathrm{d} t}^{\mathrm{sin}} \approx S_{\mathrm{d} t}^{\mathrm{tan}}$. In other words, if $\Omega_{\mathrm{c}} \ll 1$ frequency responses 
for the sine and tan filter are similar as shown in Fig. 5, where $3 \mathrm{~dB}$ cutoff frequency is $\Omega_{\mathrm{c}}=0.15 \pi$.

The sine filter compared to the tan one (i.e. standard approximation) proves to be more economical if narrow-band lowpass filter is to be designed, due to a lower hardware requirements for implementation: $n$ multipliers less needed, and $n$ three-input one-output adders replaced with standard $n$-bit carry propagate adder.

\subsection{Comparison with Other Systems}

The frequency response of the proposed tan filter (with $k=2$ and $n=8$ ) is compared with those of various 8thdegree filters derived from the product of orthogonal polynomials: Chebyshev, Legendre and transitional ButterworthChebyshev (TBC) as shown in Fig. 6. The attenuation at the passband edge frequency, $\Omega_{\mathrm{c}}=0.3 \pi$, equals to $3 \mathrm{~dB}(\varepsilon=1)$ for all approximations. The tradeoff between the maximum pass-band ripple and the group delay deviation can be noticed.

The passband magnitude frequency responses of filters derived from product of Legendre, Gegenbauer and Butterworth-Chebyshev polynomials exhibit the attenuation whose maxima is increasing function of frequency, while filter derived from product of Chebyshev polynomials exhibit the attenuation whose maxima is decreasing function of the frequency. Note that if the attenuation maxima are a decreased function of frequency in the passband, the sensitivity is also decreased. The minimum amount of maximum passband ripple corresponds to a filter derived from the product of Legendre polynomials. However, cutoff slope of TBC filter (132.87) is greater than cutoff slope of proposed (116.37), Chebyshev (118.16) and Legendre (83.91) filters.

The group delay characteristics of these filters are also shown in Fig. 6. The group delay of the proposed filter is similar to a group delay of filter derived from the product of Chebyshev polynomials. Increasement of $v_{2}$ leads to a reduction of group delay peak value.

\section{Conclusion}

A new type of continuous- and discrete-time filters using products of two Gegenbauer polynomials are investigated in this paper. A number of known filter approximations can be also obtained by the specifications of the order and degree of the Gegenbauer polynomials.

The fact that the proposed filter has two degrees of freedom, i.e. order of Gegenbauer polynomials can be adjusted (keeping the same filter degree) to meet specific requirements, proves proposed filter to be a suitable alternative to many classical choices. These free parameters allow one to obtain continuous smoothing of the magnitude frequency response in the passband compared to Chebyshev and higher cutoff rate compared to Butterworth filter.
The passband and stopband performances of the discrete-time sine and tan filters have been studied. It is shown that the tan filter is superior in comparison to both continuous-time and discrete-time sine filter. The cutoff slope depends on the cutoff frequency and increases in the case of the tan filter, decreases in the case of the sine filter, as the cutoff frequency approaches half the sampling frequency. However, for narrow-band lowpass filter the sine filter solution is better solution because its implementation requires less hardware.

\section{Acknowledgments}

This work was supported and funded by the Serbian Ministry of Education, Science and Technological Development under the project No. 32009TR.

\section{References}

[1] LAIPERT, M., VLCEK, M., VRBATA, J. Contribution to the Chebyshev approximations of the normalized low-pass prototype. Radioengineering, 2004, vol. 13 , no. 1, p. 32-36. ISSN: 1805-9600

[2] CHRYSSOMALLIS, M. T., SAHALOS, J. N. Filter synthesis using products of Legendre polynomials. Electrical Engineering, 1999, vol. 81, no. 6, p. 419-424. DOI: 10.1007/BF01387163

[3] PAI, K. R., MURTHY, K. V. V., RAMACHANDRAN, V. Chebyshev-family of transitional filters. Journal of Circuits, Systems and Computers, 1998, vol. 8, no. 2, p. 283-299. DOI: $10.1142 / \mathrm{S} 0218126698000122$

[4] BUDAK, A., ARONHIME, P. Transitional Butterworth-Chebyshev filters. IEEE Transactions on Circuit Theory, May 1971, vol. 18, no. 5, p. 413-415. DOI: 10.1109/TCT.1971.1083276

[5] ROY, S. C. D. Modified Chebyshev lowpass filters. International Journal of Circuit Theory and Applications, 2010, vol. 38, no. 5, p. 543-549. DOI: $10.1002 /$ cta.585

[6] ĆIRIĆ, D., PAVLOVIĆ, V. Generalised Christoffel-Darboux formula most directly applied in generating fully symmetric doubly resistively terminated 1c lossless ladder filters. International Journal of Electronics, 2013, vol. 100, no. 7, p. 942-958. DOI: $10.1080 / 00207217.2012 .727352$

[7] STAMENKOVIĆ, N., STOJANOVIĆ, V. On the design transitional Legendre-Butterworth filters. International Journal of Electronics Letters, 2014, vol. 2, no. 3, p. 186-195. DOI: $10.1080 / 00207217.2014 .894138$

[8] STOJANOVIĆ, N., STAMENKOVIĆ, N., STOJANOVIĆ, V. Allpole recursive digital filters design based on ultraspherical polynomials. Radioengineering, 2014, vol. 23, no. 3, p. 949-954. ISSN: 1805-9600

[9] HAZRA, S., ROY, S. C. D. A comparison of digital tan and sine filters with the generating analog filter. Journal of the Franklin Institute, 1971, vol. 292, no. 3, p. 225-230. DOI: 10.1016/0016-0032(71)90054-8

[10] ABRAMOWITZ, M., STEGUN, I. Handbook of Mathematical Functions with Formulas, Graphs, and Mathematical Tables, National Bureau of Standards Applied Mathematics Series 55, New York (USA), 1972. 
[11] ZAPLATILEK, K., LARES, M. Efficient algorithms of direct and inverse first-order $s-z$ transformations. Radioengineering, 2001, vol. 10, no. 1, p. 6-10. ISSN: 1805-9600

[12] RADER, C., GOLD, B. Digital filter design techniques in the frequency domain. Proceedings of the IEEE, 1967, vol. 55, no. 2, p. 149171. ISSN: 0019-9219

\section{About the Authors ...}

Nikola STOJANOVIĆ was born in 1973. He received his M.Sc. degree in Electronics and Telecommunication at the Faculty of Technical Sciences, University of Priština, K. Mitrovica in 1997, and M.Sc. degree in Multimedia Technologies at University of Niš at 2013. Currently he works as a lecturer of multimedia and 3D animation at Faculty of Electronics, University of Niš and a PhD student at department of Electronics at the same University.
Negovan STAMENKOVIĆ was born in 1979. He received the M.Sc. degree from the Department of Electronics and Telecommunication at the Faculty of Technical Sciences, University of Priština, K. Mitrovica in 2006 and the Ph.D. degree in electrical and computer engineering from the Faculty of Electronic Engineering, Nišs, Serbia, in 2011. He is assistant professor at Faculty of Natural Sciences and Mathematics, University of Pristina.

Ivan KRSTIĆ was born in 1988. He received the B.Sc. and M.Sc. degrees from the Department of Electronics and Telecommunication at the Faculty of Technical Sciences, University of Priština, K. Mitrovica, in 2011. and 2012. From 2013, he is working as a research assistant at Faculty of Technical Sciences, University of Priština. He is a PhD student on Faculty of Electronic Engineering, University of Niš, Serbia. 